\title{
Delayed diaphragmatic herniation masquerading as a complicated parapneumonic effusion
}

\author{
John Tsang MD FRCPC, Frank Ryan MB FRCPC \\ Divisions of Critical Care and Respiratory Medicine, Department of Medicine, \\ University of British Columbia, Vancouver, British Columbia
}

\begin{abstract}
J Tsang, F Ryan. Delayed diaphragmatic herniation masquerading as a complicated parapneumonic effusion. Can Respir J 1999;6(4):361-366.
\end{abstract}

Injury to the diaphragm following blunt or penetrating thoracoabdominal trauma is not uncommon. Recognition of this important complication of trauma continues to be a challenge because of the lack of specific clinical and plain radiographic features, the frequent presence of other serious injuries and the potential for delayed presentation. Delayed diaphragmatic herniation often presents with catastrophic bowel obstruction or strangulation. Early recognition of diaphragmatic injury is required to avoid this potentially lethal complication. The case of a 35-year-old man with a history of a knife wound to the left flank 15 years previously, who presented with unexplained acute hypoxemic respiratory failure and a unilateral exudative pleural effusion that was refractory to tube thoracostomy drainage, is reported. After admission to hospital, he developed gross dilation of his colon; emergency laparotomy revealed an incarcerated colonic herniation into the left hemithorax. Interesting clinical features of this patient's case included the patient's hobby of weightlifting, a persistently deviated mediastinum despite drainage of the pleural effusion and deceptive pleural fluid biochemical indices.

Key Words: Acute ileus; Bowel obstruction; Deviated mediastinum; Pleural effusion; Weightlifting

\section{Confusion entre épanchement parapneumoni- que compliqué et hernie diaphragmatique tar- dive}

RÉSUMÉ : L'atteinte diaphragmatique n'est pas rare après un traumatisme thoraco-abdominal fermé ou pénétrant. La reconnaissance de cette importante complication des traumatismes continue de représenter un défi, compte tenu de l'absence de caractéristiques spécifiques sur les plans des symptômes cliniques et des clichés radiologiques simples, de la présence fréquente d'autres lésions graves et du risque que les manifestations apparaissent tardivement. L'hernie diaphragmatique tardive s'accompagne souvent d'une dangereuse obstruction ou strangulation intestinale. Le diagnostic précoce de cette lésion diaphragmatique est nécessaire pour éviter une complication potentiellement fatale. Le cas présenté ici est celui d'un homme de 35 ans ayant subi 15 ans auparavant une blessure à l'arme blanche au côté gauche; il se présente pour insuffisance respiratoire hypoxémique aiguë inexpliquée et épanchement pleural exsudatif bilatéral réfractaire au traitement par thoracotomie. Après son admission à l'hôpital, il a présenté une dilatation marquée du côlon. La laparotomie d'urgence a révélé une hernie irréductible du côlon dans l'hémithorax gauche. Parmi les caractéristiques intéressantes de ce cas, mentionnons le passetemps du patient, haltérophile, un médiastin présentant une déviation persistante malgré le drainage de l'épanchement pleural et les indices biochimiques trompeurs du liquide pleural.

Correspondence and reprints: Dr John Tsang, Pulmonary Research Laboratory, University of British Columbia, 1081 Burrard Street, Vancouver, British Columbia V6Z 1Y6. Telephone 604-878-0257, fax 604-524-9623, e-mail jtsang@interchange.ubc.ca 
$\mathrm{T}$ raumatic injury and subsequent herniation through the diaphragm was first described over 400 years ago (1), and the first successful repair was reported in 1886 (2). Correct diagnosis of this potentially lethal condition continues to require a high index of suspicion (3). Surgical intervention must be provided promptly because the patient's clinical course may rapidly deteriorate with bowel strangulation, obstruction, hemorrhage and perforation, as well as empyema and pleural fistula (4). The exact incidence of delayed diaphragmatic hernia occurring many years after the initial trauma is not well documented in the literature, although this complication is well recognized. We present a case of a young man who developed acute hypoxemic respiratory failure, a persistent left-sided pleural effusion and sudden onset of ileus 15 years after a stab wound to the left upper quadrant of his abdomen.

\section{CASE PRESENTATION}

A 35-year-old man presented to the emergency room complaining of a two-day history of left-sided chest pain in the axillary area. The pain awoke him from sleep the morning after a party at which he had consumed a large amount of alcohol. The pain persisted despite position changes and oral analgesics, and he eventually sought medical attention when his dyspnea at rest worsened. He complained of a mild sore throat and vague abdominal pain in the preceding few days, but denied a recent history of fever, chills, increasing cough or sputum production. Past history revealed cigarette consumption of one pack/day for the previous 15 years and occasional cocaine use by inhalation. He used anabolic steroids intramuscularly to enhance his weightlifting and body building performance. Of note was a history of a superficial knife

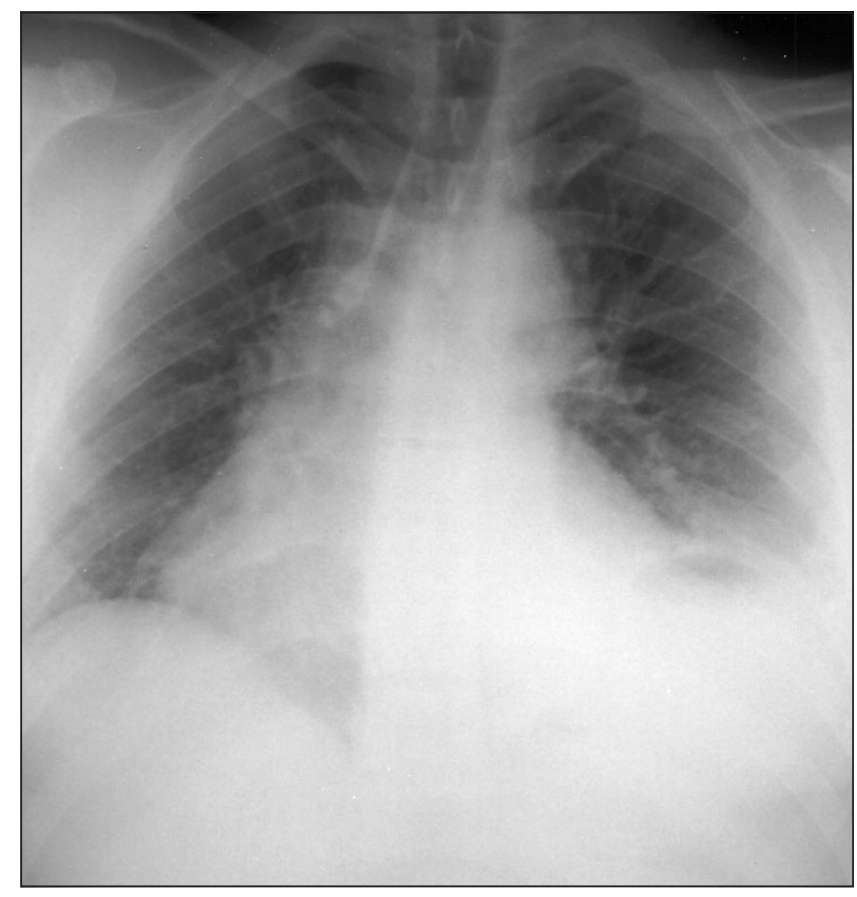

wound to the left upper quadrant of the abdomen 15 years previously. The significance of this injury was discounted initially because it was reported to be a superficial laceration that was sutured, followed by prompt discharge from the emergency room.

Physical examination revealed a muscular young man weighing approximately $100 \mathrm{~kg}$, in moderate respiratory distress, lying on his right side. He was alert and orientated, temperature was $37.9^{\circ} \mathrm{C}$, blood pressure $136 / 70 \mathrm{mmHg}$, heart rate 100 beats/min in sinus rhythm and a respiratory rate of 36 breaths/min, shallow with splinting. Head and neck examination showed an erythematous pharynx suggestive of a recent upper respiratory tract infection. Mucous membranes were dry, and jugular venous pressure was $2 \mathrm{~cm} \mathrm{H}_{2} \mathrm{O}$. Chest expansion was reduced on the left side, with decreased tactile fremitus, dullness to percussion and decreased air entry to the left base. Heart sounds were normal. There was mild generalized guarding in the abdomen but no peritoneal signs. Bowel sounds were present. An old healed $4 \mathrm{~cm}$ scar was noted below the left costal margin. The remainder of the physical examination was unremarkable; there was no evidence of recent trauma, deep vein thrombosis or intravenous needle track marks.

Laboratory data revealed hemoglobin of $163 \mathrm{~g} / \mathrm{L}$ and white blood cell (WBC) count of $24.6 \times 10^{9} / \mathrm{L}$, with a neutrophil count of $21 \times 10^{9} / \mathrm{L}$. Arterial blood gases on room air showed a $\mathrm{pH}$ of 7.44 , partial pressure of oxygen $82 \mathrm{mmHg}$, partial pressure of carbon dioxide $41 \mathrm{mmHg}$, and bicarbonate $28 \mathrm{mmol} / \mathrm{L}$. Biochemical renal function was normal. An electrocardiogram showed sinus tachycardia and no acute ischemic changes. The admission chest radiograph revealed elevation and partial obscuration of the left hemidiaphragm,

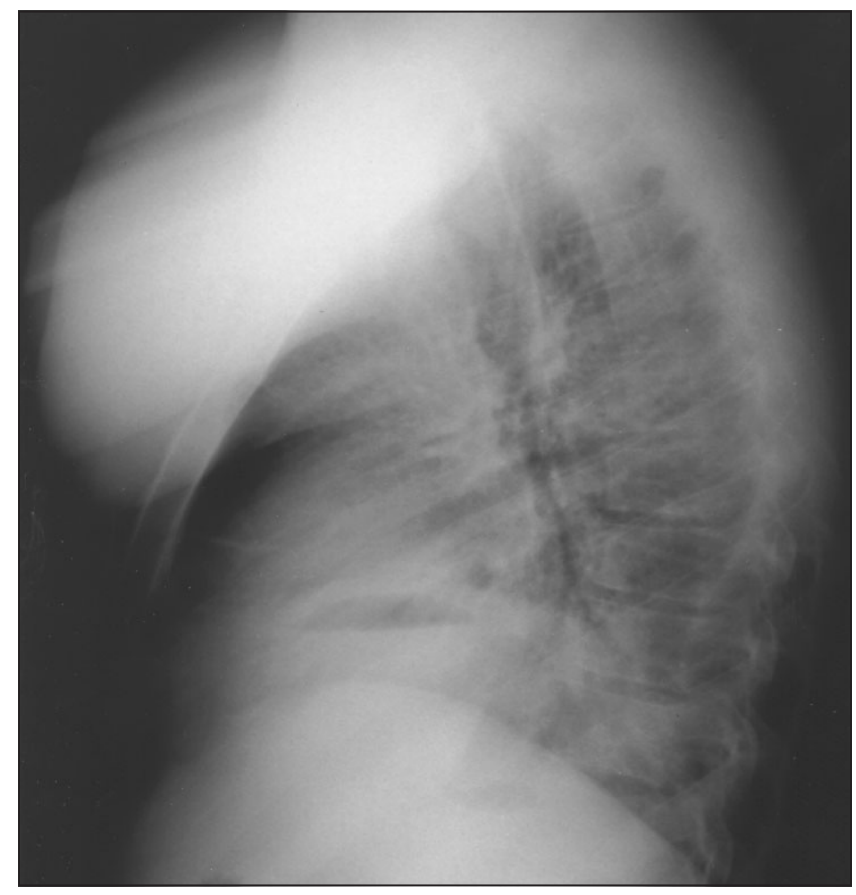

Figure 1) Admission chest radiographs showing elevation and partial obscuration of the left hemidiaphragm and mediastinal shift to the right. An air fluid level appears to lie beneath the diaphragm, possibly in the stomach. On the lateral view (right), air bronchograms and obscuration of the posterior hemidiaphragm were interpreted as evidence of consolidation in the left lower lobe 

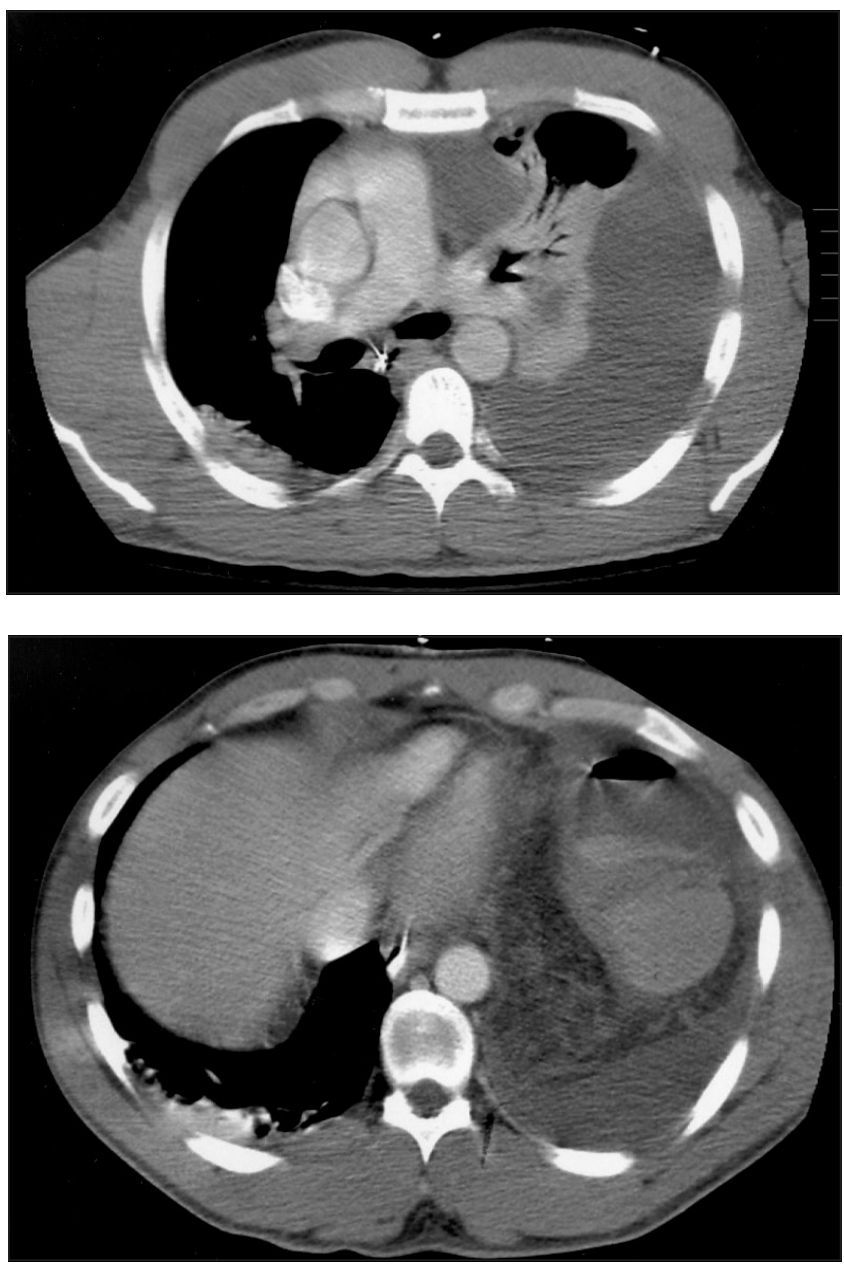

Figure 2) Computed tomographic images of the chest (mediastinal windows). Top A large left pleural effusion, atelectasis of the left lower lobe and mediastinal shift to the right are apparent. Bottom An air-fluid level is visible and was felt to be in a loop of bowel beneath the left hemidiaphragm

air bronchograms at the left base, and mediastinal shift to the right (Figure 1).

The patient was admitted to the medical ward with a presumptive diagnosis of community-acquired left lower lobe pneumonia. After a septic work-up was completed, he was treated with oxygen, intravenous fluid and empirical antibiotics including erythromycin and cefuroxime, pending the result of sputum culture and sensitivity. A ventilation perfusion scan indicated low probability for pulmonary embolism.

Over the next $18 \mathrm{~h}$, the patient's dyspnea continued to worsen with increasing use of accessory muscles, and his oxygen requirements by mask increased from $40 \%$ to $90 \%$ oxygen in order to maintain oximetric oxygen saturation above $94 \%$. A left-sided pleural rub was heard. Arterial blood gases on $100 \%$ oxygen revealed $\mathrm{pH} 7.40$, partial pressure of oxygen $164 \mathrm{mmHg}$, and partial pressure of carbon dioxide $43 \mathrm{mmHg}$ (ie, estimated alveolar-arterial oxygen gradient about 500 and partial pressure of arterial oxygen to forced inspiratory oxygen ratio of 164). The patient was transferred to the intensive care unit (ICU), and he was stabilized by intubation and mechanical ventilation. A repeat
TABLE 1

Pleural fluid analysis from patient with delayed diaphragmatic hernia

\begin{tabular}{lc}
\hline Test & Result \\
\hline Hematology & \\
Red blood cells & $192,000 \times 10^{6} / \mathrm{L}$ \\
White blood cells & $17,500 \times 10^{6} / \mathrm{L}$ \\
Neutrophils & $100 \%$ \\
Biochemistry & \\
$\mathrm{pH}$ & 7.0 \\
Glucose & $<0.3 \mathrm{mMol} / \mathrm{L}$ \\
Protein & $47 \mathrm{~g} / \mathrm{L}$ \\
Lactate dehydrogenase & $1473 \mathrm{U} / \mathrm{L}$ \\
Amylase & $<30 \mathrm{mmol} / \mathrm{L}$ \\
Microbiology & \\
Gram stain & 1. 2+ polymorphonuclear neutrophil \\
& 2. Occasional Gram-negative rods \\
& 3. Occasional Gram-positive cocci \\
\hline
\end{tabular}

chest radiograph showed an enlarging left pleural effusion and persistent mediastinal shift to the right despite assist control positive pressure ventilation. Left lower lobe atelectasis and consolidation were again noted. His fever worsened, and peripheral WBC count rose to $32.1 \times 10^{9} / \mathrm{L}$. Erythromycin was increased to $1 \mathrm{~g}$ intravenous every $6 \mathrm{~h}$, cefuroxime was discontinued and piperacillin $3 \mathrm{~g}$ intravenous every $6 \mathrm{~h}$ was substituted. High doses of morphine and midazolam were required to maintain sedation. Enteral feeding via nasogastric feeding tube was commenced without difficulty.

A computed tomographic (CT) scan of the chest confirmed a large left pleural effusion, shift of the mediastinal structures to the right and consolidation/atelectasis of the left lower lobe. A small air-fluid level separate from the stomach was identified but attributed to a loop of bowel beneath an elevated left hemidiaphragm (Figure 2). The mediastinal deviation was attributed to the large left pleural effusion. A diagnostic thoracentesis yielded bloodstained pleural fluid, an analysis that was consistent with a complicated parapneumonic effusion (5) (Table 1). A tube thoracostomy performed under local anaesthesia yielded $1200 \mathrm{~mL}$ of pleural fluid over $24 \mathrm{~h}$. Subsequently, streptokinase was instilled into the pleural space to enhance pleural fluid drainage (6).

Over the next three days, the patient's condition remained critical and he was unable to tolerate attempts to wean him from ventilatory support. Serial chest radiographs showed minimal improvement despite aggressive drainage therapy (Figure 3). On the fourth hospital day, nasogastric tube feeding was stopped because of a sudden increase in gastric residuals and progressive abdominal distension. Physical examination revealed new findings of generalized tenderness and tympanic percussion in the abdomen. A flat plate abdominal radiograph showed gross colonic dilation with the diameter ranging from 7 to $10 \mathrm{~cm}$ in the cecum and transverse colon, but residual air in the sigmoid colon was also noted. Acute adynamic ileus was felt to be unlikely because the pa- 


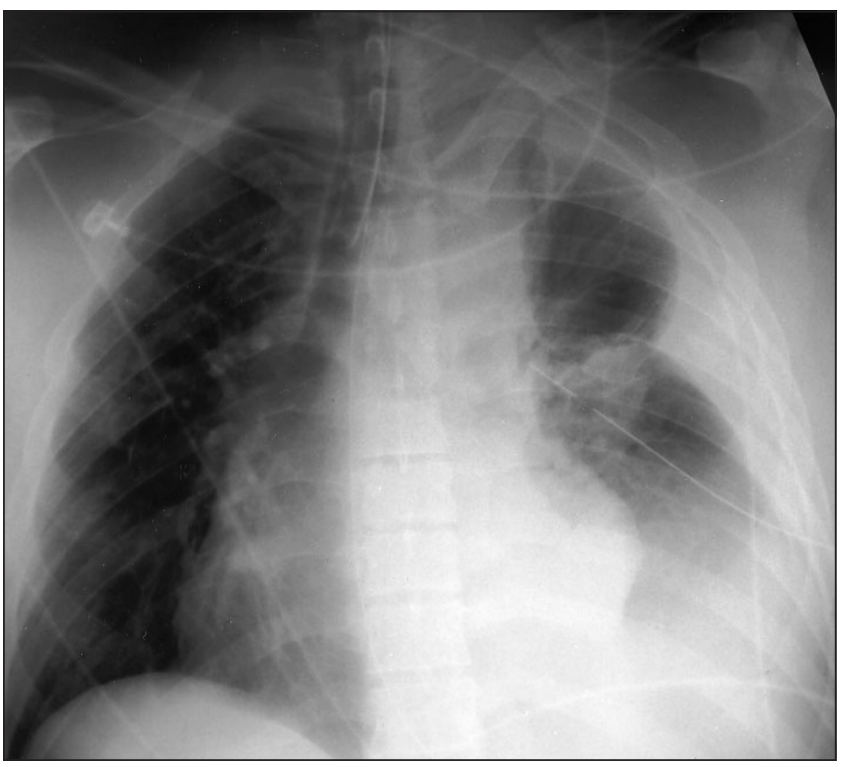

Figure 3) A portable chest radiograph after left tube thoracostomy shows persistent mediastinal shift, left pleural effusion, complete obscuration of the left hemidiaphragm and no evidence of an intrathoracic air-fluid level

tient was receiving high doses of erythromycin, which is known to promote gastrointestinal (GI) tract motility (7).

An emergency colonoscopy was performed. At approximately $80 \mathrm{~cm}$ from the anal verge, a stricture containing macerated and erythematous mucosa was encountered, beyond which the scope could not be passed. Because of the impending risk of bowel perforation and with no prospect of conservative decompression, a surgical consultation was sought and an immediate exploratory laparotomy was scheduled. Metronidazole and gentamicin were commenced intravenously before surgery. Culture of the pleural fluid later grew Enterococcus fecalis and Escherichia coli.

Intraoperatively, massive dilation of the right and transverse colon was confirmed. In addition, there was a $5 \mathrm{~cm}$ perforation in the cecum, which was adherent to the small bowel, and an area of ischemia in the transverse colon. Despite careful manipulation, this necrotic segment perforated intraoperatively and caused gross fecal contamination. Further exploration revealed herniation of approximately $30 \mathrm{~mL}$ of colon at the splenic flexure into the left hemithorax through a $3 \mathrm{~cm}$ defect in the diaphragm. The herniated bowel appeared necrotic, and no attempt was made to reduce it at this stage. A left thoracotomy was then performed, and the necrotic segment of colon was reduced into the abdomen through an extended incision in the diaphragm. A decortication was carried out, and the diaphragmatic defect was closed with 15 interrupted 0 -silk sutures. After vigorous irrigation of the abdominal cavity, a subtotal colectomy and ileostomy were performed and a mucous fistula was brought up from the descending colon to the inferior aspect of the abdominal wound. Perioperatively, the patient remained hemodynamically stable with good urine output throughout.

A postoperative chest radiograph showed an essentially

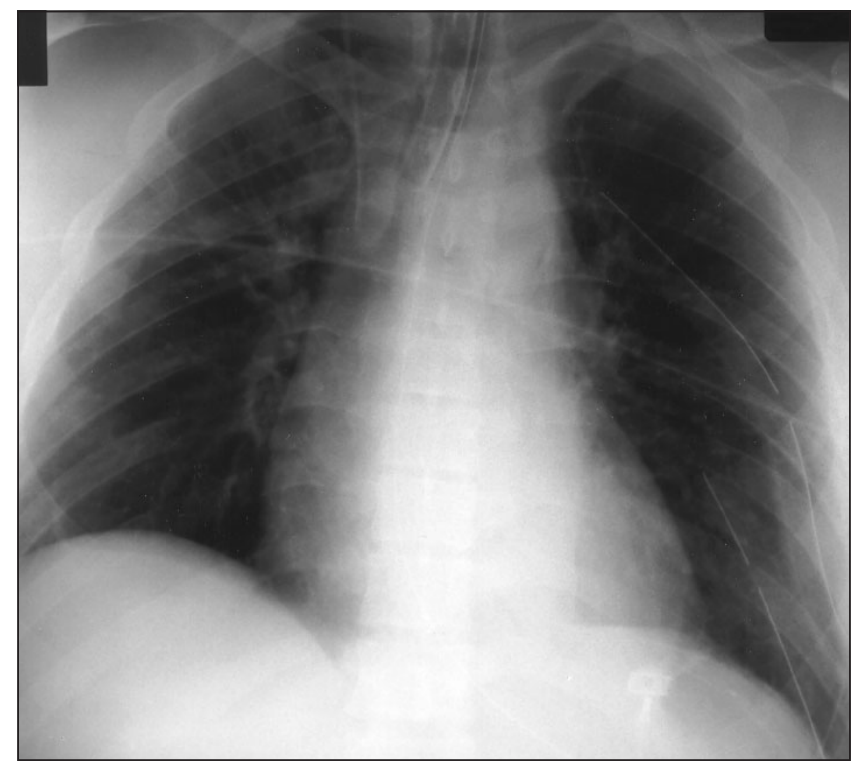

Figure 4) Immediate postoperative chest radiograph showing essentially normal left lung, pleural space and hemidiaphragm. The mediastinum returned toward the midline

normal left lung and hemidiaphragm, and the mediastinal structures had essentially returned to the midline (Figure 4). Further review of the chest CT scan confirmed that the previously noted air-fluid level was in fact a loop of colon within the left hemithorax.

The patient's clinical course continued to improve rapidly after surgery, and he was extubated three days postoperatively without ever requiring vasopressor support. He was discharged from the ICU and remained in the hospital for a further 12 days of convalescence before being discharged home in good condition. He subsequently underwent an uneventful secondary reanastomosis of his bowel.

\section{DISCUSSION}

This case illustrates the difficulty in diagnosing delayed diaphragmatic herniation. Several interesting features of this case merit further discussion, including the long interval between the original injury and the development of a diaphragmatic hernia, and the possible role of weightlifting in its occurrence; the misleading pleural fluid biochemistry; the persistently deviated mediastinum; and the development of ileus in a critically ill patient.

Delayed presentation of post-traumatic diaphragmatic hernia: An earlier report documented one patient who presented with diaphragmatic hernia 44 years after the initial injury (8). Because of the persistently positive pressure gradient of 7 to $20 \mathrm{~cm} \mathrm{H}_{2} \mathrm{O}$ between the peritoneal and pleural cavities, any defect in the diaphragm tends not to close spontaneously and indeed becomes enlarged over time $(9,10)$. During the maximal straining of a Valsalva manoeuvre such as weightlifting, the pleuroperitoneal pressure gradient can reach $45 \mathrm{~cm} \mathrm{H}_{2} \mathrm{O}$ (11). Repeated exposures to this kind of force can promote transdiaphragmatic herniation of any sus- 
ceptible abdominal viscera such as the colon and stomach. Interestingly, some motor vehicle accident victims with ruptured diaphragms have claimed to be aware of the impending crash and tightened their abdominal muscles in a Valsalva manoeuvre, leaving the diaphragm as the weakest point of pressure release upon external impact (12). When such a crush injury occurs in the trunk, the pleuroperitoneal pressure gradient upon external impact dramatically increases beyond its already higher baseline level from the Valsalva manoeuvre.

A review of several case series reveals that, in patients with penetrating or blunt trauma, hemidiaphragmatic herniation occurs about 2.5 times more commonly on the left (13), although right-sided injuries are being seen with increasing frequency (14). The reasons for this difference include greater muscular bulk of the right hemidiaphragm, buffering effect by the liver on the right side, the weaker embryonic fusion points on the left (15) and underdiagnosis of right-sided injury in the past. In the case of blunt trauma such as motor vehicle accidents, left-sided impact poses a greater risk of diaphragmatic rupture than either frontal or right-sided impact (12). Deformation shear of the stretched diaphragm is probably the mechanism of rupture (16).

The location of our patient's diaphragmatic hernia is no doubt related to the remote stab wound in the left lower costal margin. For delayed rupture, surgical reduction of herniated abdominal viscera and repair of the hemidiaphragm through a thoracotomy incision is the treatment of choice, because adhesions to the lung, pericardium and mediastinum are invariably present (17). A layer of synthetic mesh is sometimes used for reinforcement provided that the operative field is not contaminated (18).

Misleading pleural fluid biochemistry: While the biochemical characteristics of the pleural fluid obtained at thoracentesis were compatible with a complicated parapneumonic effusion (5), the presence of a bloody effusion was atypical and should have raised the suspicion of some other process such as an ischemic viscus. The possibility of esophageal perforation was considered, but the low amylase level did not support this diagnosis. In retrospect, the low amylase was not inconsistent with the eventual diagnosis because it was measured early in the patient's course, probably before the incarcerated bowel became infarcted. It was unclear whether the pleural fluid had been derived from the visceral pleura, the incarcerated bowel or the peritoneal cavity.

Persistently deviated mediastinal structures: The significance of the persistently shifted mediastinal structures to the right was not appreciated and was incorrectly attributed to a tension hydrothorax secondary to a parapneumonic effusion. Most such effusions, however, would be expected to resolve or improve significantly after chest tube drainage, with or without streptokinase instillation (6). Although mediastinal shift to the contralateral side has been reported in association with hemidiaphragm rupture (19), this important sign has not been particularly emphasized, nor has its mechanism been discussed in detail. The mediastinal shift may relate to positive pressure in the ipsilateral hemithorax due to the herni- ated abdominal contents. Alternatively during spontaneous breathing, it may be due to ineffective negative pressure generation by the ruptured hemidiaphragm. In the latter situation, a chest radiograph taken in expiration might show a return of the mediastinum towards the midline.

Among other plain radiographic clues to the diagnosis of diaphragmatic herniation, the finding of the tip of a nasogastric tube in the hemithorax is only useful if the stomach is the herniated viscus, which was not the case in our patient. The presence of an air-fluid level above an elevated hemidiaphragm is an important sign, which only appeared late in our patient's course. The available investigative techniques include a chest $\mathrm{x}$-ray with or without nasogastric tube in place, contrast studies, ultrasonography, radioisotopic scintigraphy of the liver and spleen (20), CT scan (21), thoracoscopy, laparoscopy, and peritoneal lavage (22). The least invasive investigations are more commonly used initially. Pathognomonic signs are gas bubbles or nasogastric tube in the chest, or a positive Gastrografin study. Irregularity or elevation of the diaphragmatic contour, atelectasis of the lower lobe or contralateral mediastinal shift without a well-defined pulmonary pathology are certainly suggestive, although less specific.

Onset of GI signs and symptoms after hypoxemic respiratory failure: Enteral feeding was initiated early without difficulty after the patient was intubated in the ICU, at which time the abdominal examination was unremarkable. Acute ileus then developed unexpectedly three days later despite the concomitant use of high dose erythromycin, which enhances GI motility (7). The persistent left-sided chest pain radiating to the axilla and shoulder suggests that there was diaphragmatic herniation early on, but without bowel obstruction initially. Before the final stage of strangulation and venous infarction of the herniated colon, bowel movements continued to some extent and gas was observed in the sigmoid colon. Because the patient could not communicate verbally on account of endotracheal intubation and heavy sedation, a sudden increase in the volume of the gastric residuals during continuous enteral feeding was an important sign of bowel obstruction in a patient who had a recent or remote history of diaphragmatic injury.

\section{CONCLUSIONS}

With the benefit of hindsight, the sequence of events in this case appears somewhat clearer now. We suspect the stab wound in the left upper quandrant was the cause of this patient's diaphragmatic injury. Heavy weightlifting may have further contributed to enlargement of the defect, with resultant delayed bowel herniation many years later. The apparent elevation of the left hemidiaphragm in association with the contralateral mediastinal shift should have alerted us to the possibility of diaphragm injury. The bloody appearance of the pleural fluid should have been accorded more significance, rather than relying on the biochemical analysis. The subsequent culture result of $E$ fecalis and $E$ coli in the pleural fluid came too late to alert us to a possible bowel source. 
ACKNOWLEDGEMENTS: The authors thank Drs Barbara Heller, Alan Weiss and Richard Finley for their concurrent care of this patient.

\section{REFERENCES}

1. Schneider CF. Traumatic diaphragmatic hernia. Am J Surg 1956;91:290-4.

2. Hedblom CA. Diaphragmatic hernia. JAMA 1925;85:947-53.

3. Troop B, Myers RM, Agarwal NN. Early recognition of diaphragmatic injuries from blunt trauma. Ann Emerg Med 1985;14:97-101.

4. Feliciano DV, Cruse PA, Mattox KL, et al. Delayed diagnosis of injuries to the diaphragm after penetrating wounds. J Trauma 1988;28:1135-44.

5. Light RW. Parapneumonic effusion and empyema. In: Light RW ed. Pleural Diseases, 3rd edn. Baltimore: Williams \& Wilkins, 1995:129-53.

6. Davies RJO, Traill ZC, Gleeson FV. Randomized controlled trial of intrapleural streptokinase in community acquired pleural infection. Thorax 1997;52:416-21.

7. Dive A, Miesse C, Galanti L, et al. Effect of erythromycin on gastric motility in mechanically ventilated critically ill patients: a double blind, randomized, placebo-controlled study. Crit Care Med 1995;23:1356-62.

8. Epstein LI, Lempke RE. Rupture of the right hemidiaphragm due to blunt trauma. J Trauma 1968;8:19-28.

9. Shah RS, Sabanathan S, Mearns A, Choundhury AK. Traumatic rupture of diaphragm. Ann Thorac Surg 1995;60:1444-9.

10. Waldschmidt ML, Laws HL. Injuries of the diaphragm. J Trauma 1980;20:587-92.
11. Marchand P. A study of the forces productive of gastro-oesphageal regurgitation and herniation through the diaphragmatic hiatus. J Trauma 1957;12:189-202.

12. Kearney PA, Rouhana SW, Burney RE. Blunt rupture of the diaphragm: Mechanism, diagnosis, and treatment. Ann Emerg Med 1989;18:1326-30.

13. Sharma OP. Traumatic diaphragmatic rupture: Not a uncommon entity - personal experience with collective review of the 1980's. J Trauma 1989;29:678-82.

14. Jarret F, Bernhardt LC. Right-sided diaphragmatic injury. Arch Surg 1978;113:737-9.

15. Mansour KA. Trauma to the diagphragm. Chest Surg Clin North Am 1997;7:373-83.

16. Ward RE, Flynn TC, Clark WP. Diaphragmatic disruption secondary to blunt abdominal trauma. J Trauma 1981;21:25-8

17. Hill LD. Injuries of the diaphragm following blunt trauma. Surg Clin North Am 1972;52:611-24.

18. Waldhausen JA, Kilman JW, Helman CH, Battersby JS. The diagnosis and management of traumatic injuries of the diaphragm including the use of marlex prostheses. J Trauma 1966;6:332-43.

19. Felson B. Chest Roentgenology. Philadelphia: WB Saunders, 1973:437.

20. Kim EE, McConnell BJ, McConnell RW, Duke JH, Dillon M. Radionuclide diagnosis of diaphragmatic rupture with hepatic herniation. Surgery 1983;94:36-40.

21. Heiberg E, Wolverson MK, Hurd RN, Jagannadharao B, Sundaram M. CT recognition of traumatic rupture of the diaphragm. AJR Am J Roentgenol 1980;135:369-72.

22. Van Vugt AB, Schoots FJ. Acute diaphragmatic rupture due to blunt trauma: a retrospective analysis. J Trauma 1989;29:683-6. 


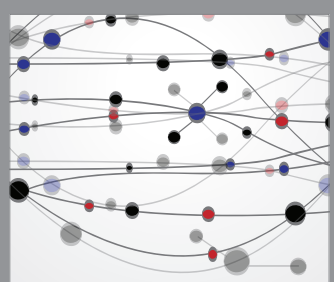

The Scientific World Journal
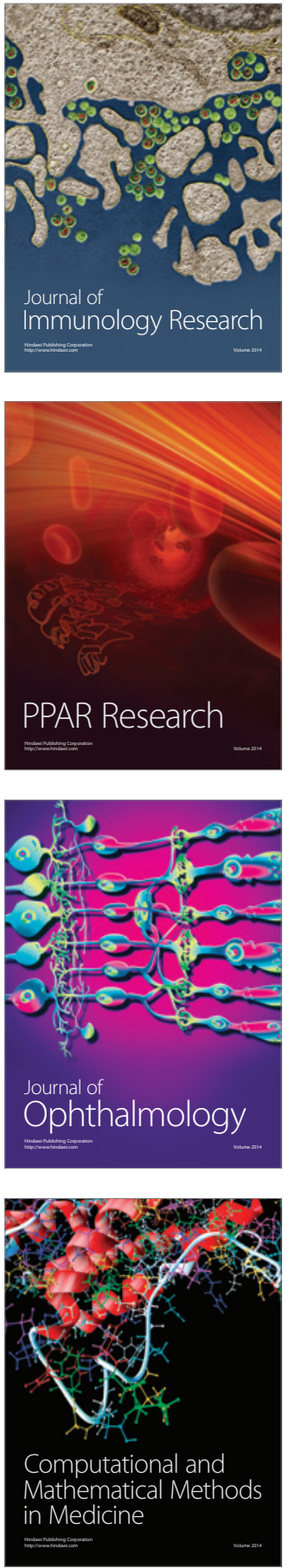

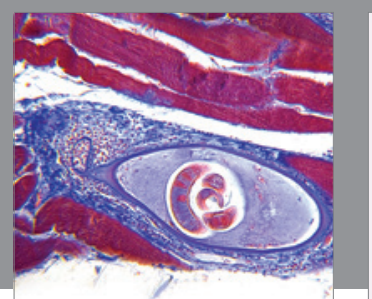

Gastroenterology Research and Practice

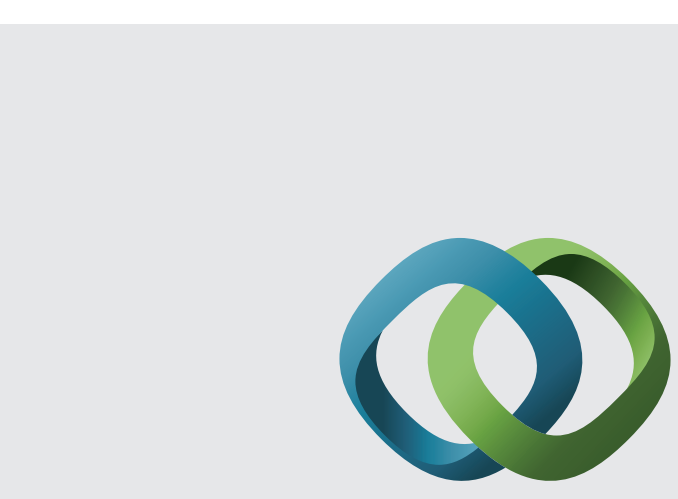

\section{Hindawi}

Submit your manuscripts at

http://www.hindawi.com
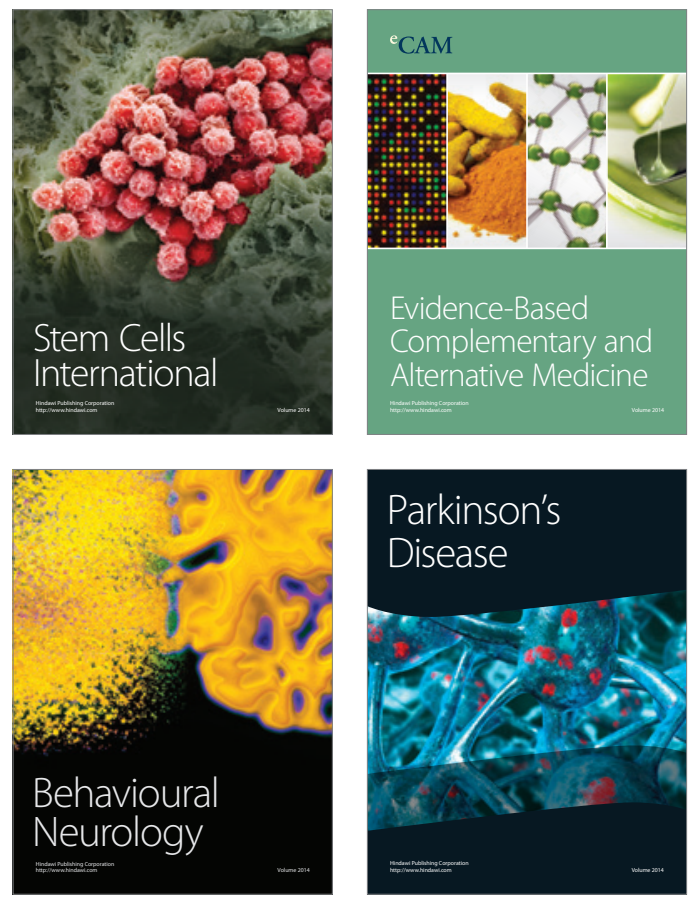
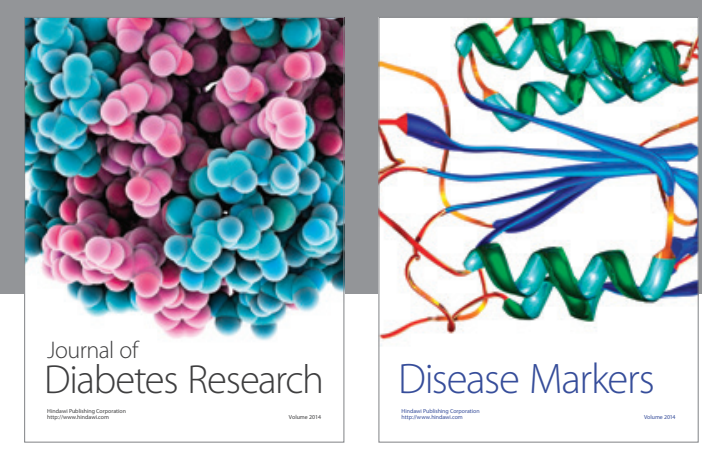

Disease Markers
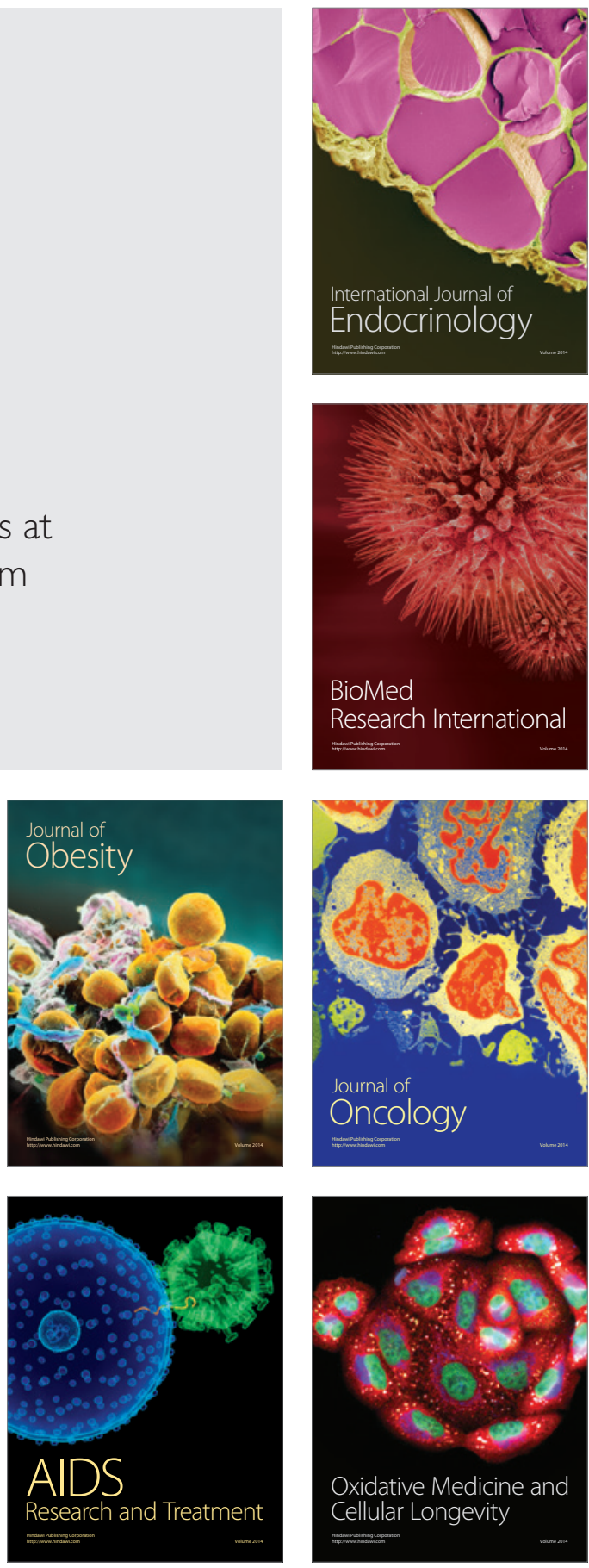